\title{
Enquête
}

Archives de la revue Enquête

$8 \mid 1993$

Varia

\section{Portrait de Richard Hoggart en sociologue}

Jean-Claude Passeron

\section{(2) OpenEdition \\ Journals}

Édition électronique

URL : http://journals.openedition.org/enquete/175

DOI : 10.4000/enquete. 175

ISSN : 1953-809X

Éditeur :

Cercom, Éditions Parenthèses

Édition imprimée

Date de publication : 2 septembre 1993

Pagination : 79-111

Référence électronique

Jean-Claude Passeron, «Portrait de Richard Hoggart en sociologue », Enquête [En ligne], 8 | 1993, mis en ligne le 12 mai 2006, consulté le 19 avril 2019. URL : http://journals.openedition.org/enquete/175 ; DOI : 10.4000/enquete. 175

Ce document a été généré automatiquement le 19 avril 2019 


\title{
Portrait de Richard Hoggart en sociologue*
}

\author{
Jean-Claude Passeron
}

\begin{abstract}
Pour Raymonde Moulin, qui mettait le doigt, dès les années 60 , sur la contradiction entre mon goût personnel pour la lecture romanesque et ma prédication professorale de l'ascèse quantitativiste en sociologie.
\end{abstract}

1 L'admiration - celle du sociologue mais aussi celle du lecteur captivé par le ton du récit ou le travail de mémoire - que je porte à Richard Hoggart me pose une question qui me met en contradiction flagrante avec mes propos habituels sur les rapports entre l'écriture sociologique et l'écriture littéraire. Hoggart est sans doute le seul auteur qui m'oblige à me contredire à ce point et sur ce point. C'était visible dans la "Présentation » sociologisante que j'ai donnée, en 1970, de The Uses of Literacy, titre que nous avions volontairement déplacé vers la sociologie en le traduisant par La culture du pauvre ${ }^{1}$. C'est encore plus net quand je lis A Local Habitation, deuxième livre du même auteur dont les traducteurs ont préféré déplacer le titre vers le réalisme romanesque, en donnant l'adresse postale de ce « lieu d'où l'on part » : 33 Newport Street ${ }^{2}$, l'habitation d'enfance de Hoggart à Hunslet, faubourg de Leeds.

Le paradoxe-Hoggart

2 - Peut-on parler d'une position originale de cet auteur entre littérature et sociologie?

3 - À la fin des années 50, nous ne devions pas être nombreux en France à nous être entichés de The Uses of Literacy ${ }^{3}$, ce texte lisse et sans éclats de plume, concret et exhaustif, juste et informé, romanesque si l'on veut, mais par le seul souci du détail et de la nuance inlassablement poursuivis, émouvant sans aucune sensiblerie, décrivant chaleureusement mais sans lyrisme ni moralisme les valeurs, muettes ou parlantes, qui font le sens d'une culture populaire de banlieue industrielle anglaise, à la manière dont les ethnologues décrivent la cohérence de n'importe quelle autre culture étrangère à nos habitudes mentales et affectives; ce livre dépourvu de presque tous les signes extérieurs 
d'appartenance aux sciences sociales et qui, pourtant, nous semblait d'une bien meilleure sociologie des classes populaires que tout ce que nous pouvions lire dans les arides sociographies des spécialistes du chiffre ou les froides divagations idéologiques d'intellectuels «engagés ». L'éditeur français, pourtant excellent pronostiqueur des succès de la librairie parisienne, résistait. Sans doute voyait-il trop clairement qu'il ne s'agissait là $n i$ de littérature d'avant-garde $n i$ d'un pamphlet politique $n i$ de sciences humaines estampillées au sceau d'une affiliation célébrée par la critique. Pierre Bourdieu qui dirigeait la collection du "Sens commun » a tenu bon; la traduction - difficultueuse sur un texte riche en tournures familières et en détails d'une vie quotidienne exotiquement britannique en sa banalité même - était terminée (et déjà payée, ce qui est un fort argument auprès d'un éditeur). Le livre s'est fait. Contre toute attente il a été lu et a eu quelque influence en France, si j'en juge par les citations qu'en font les sociologues.

Cela fait donc longtemps que je me pose la question : «Mais sur quel pied dois-je danser quand j'admire l'écriture de Richard Hoggart?» Ce diable d'auteur me prend à contrepied, tout en me faisant valser à son rythme. J'aime - je crois - les émotions en abysme liées à la lecture littéraire. J'aime aussi les satisfactions simples et fortes de l'analyse sociologique - puisque j'en lis beaucoup et la pratique un peu. Mais les deux séparément, ayant, en la matière, une longue expérience de mes agacements de lecteur frustré simultanément du plaisir littéraire et de celui de la connaissance scientifique en tant d'ouvrages dont les titres - ou les "quatrièmes de couverture »- me promettaient les deux: ces doubles jeux n'ont souvent d'autre effet, et peut-être d'autre cause, que d'autoriser l'auteur à la double facilité de se dispenser du labeur de la recherche comme des risques de l'écriture en l'incitant à se défausser alternativement de chacune des deux exigences sur l'exigence alternative ; le lecteur, lui, y perd à tous coups son temps et sur les deux tableaux, s'il s'est laissé allécher par la prime publicitaire qui va à l'amalgame vite troussé que j'appelle «essayisme ». Le vrai « essai » est tout autre chose lorsqu'il ne se réduit pas à de la vulgarisation bien faite et parvient à associer la documentation pertinente et l'invention conceptuelle à la vivacité persuasive du style; mais peut-on encore en écrire d'honnêtes depuis que les sciences sociales ont dû renoncer, en aiguisant la pointe de leurs méthodes et en alourdissant le poids de leurs corpus, à la légèreté d'exposition qui, au XVIII siècle, seyait encore à toutes les sciences? Récemment, essayant de distinguer, dans "L'illusion romanesque ${ }^{4}$ ", entre le ressort littéraire des " effets de réel ${ }^{5}$ ", tels que les produit le roman classique, et les « effets de connaissance ", tels que les produit une méthodologie sociologique, je concluais que «le romancier inchoatif menace le sociologue plus que le sociologue préalable ne menace le romancier : on a souvent vu faire de la bonne littérature avec de la mauvaise sociologie, parfois même avec de la bonne, jamais de la bonne sociologie avec de la littérature, bonne ou mauvaise ». Et à lire aujourd'hui tant d'ouvrages qui ne feignent de pratiquer l'un des deux genres que pour se dispenser des tâches ou des difficultés de l'autre, j'en reste persuadé. Voyez combien, déjà, devenaient ennuyeux Balzac, Hugo ou Zola, quelle rupture du " pacte romanesque » produisaient, dans la littérature réaliste, les romanciers ratiocinateurs ou moralisateurs quand, intervenant directement dans le roman comme conférenciers, scholiastes, guides, tuteurs ou prophètes, ils se faisaient historiens ou sociologues du contexte de leur intrigue. Mais, indiscutablement, Hoggart échappe au champ d'application de la deuxième partie de ma phrase. Pourquoi et comment ?

5 Je ne suis pas le seul à le trouver excellent sociologue. Et à trouver d'excellente méthode - non pas seulement dans l'exposition des faits mais aussi dans le travail de 
l'interprétation - ce style et ce ton de simplicité descriptive, qui n'appartiennent qu'à lui, le distinguant même, malgré la ressemblance des objets, des autres sociologues ou anthropologues anglo-saxons de la vie populaire, pourtant plus matter-of-fact que leurs homologues continentaux dont la phrase est vite enflée ou gauchie par l'humeur politique, dès qu'il y va du " peuple ", de ses faiblesses ou de ses vertus, de ses passions ou de ses indifférences. La sociologie hoggartienne se reconnaît d'abord à sa manière de maintenir à distance les outils habituels de la distanciation scientifique comme les mots accoutumés qui font la pesanteur sociologique ou l'exotisme ethnologique des livres trop savants, tant en leurs labyrinthes empiriques (statistiques ou ethnographiques) qu'en leurs apparats théoriques (destructeurs, même quand ils semblent nécessaires, de toute légèreté de style). Chez Hoggart pas d'artillerie lourde, pas de technologie dissuasive, de polémique rituelle avec les confrères ou les ancêtres, de notes de bas de page jusqu'à plus soif. On dirait de la littérature, et de la bonne - en entendant par là du texte « lisible », doté d'harmoniques et d'échos qui "épaississent » la certitude de "comprendre » en même temps que le plaisir de "ressentir» par procuration ou fiction - mais ce n'est pas de la littérature, si l'on entend caractériser par ce mot un projet d'écriture qui serait à lui-même sa fin expressive, indépendamment de toute fonction « référentielle ${ }^{6} »$ ou argumentative : cette légèreté dans l'enchaînement narratif de la remémoration, cette richesse subtile dans l'interprétation des significations culturelles ne sont utilisées qu'à investir, qu'à servir, qu'à faire comprendre sociologiquement l'objet, un objet rebelle - la culture populaire - qui se dérobe ou se volatilise si facilement sous d'autres scalpels; ou qui se fait grimace et caricature dans les tableaux dressés par d'autres explorateurs pour les besoins d'une "bonne cause ", intellectuelle ou politique, parfois aussi pour les besoins plus crus d'un appareil ou d'une corporation avides d'annexer ou d'enrôler des fidèles.

6 Ce qu'il y a de « littéraire » dans l'écriture d'Hoggart n'altère jamais le prosaïsme et la clarté qu'exige le contrôle de la "véridicité » des assertions, but laborieux de toute analyse sociologique qui se réclame d'une méthode scientifique. Si « l'invitation au sabbat littéraire est un appel qui se fait entendre au plus intime de l'interprétation sociologique, dans l'insatisfaction même de son travail amer de production des connaissances ${ }^{7}$ » (je l'ai écrit ailleurs, pour faire remarquer que les plus arides des sociologues n'étaient pas exempts de toute faiblesse à l'égard des facilités de "l'illusion romanesque »), Hoggart ne cède jamais à l'utopie du "réalisme littéraire ${ }^{8}$ ", c'est-à-dire à la tentation toujours renaissante d'un "art scientifique ", lequel - en dépit de la revendication de tant de romanciers qui se sont voulus les " peintres » de leur société - dénature inévitablement le projet de connaissance sociologique puisqu'il doit, sans le dire, subordonner ce projet à la recherche d'un effet proprement littéraire, c'est-à-dire à la création d'un émoi inconscient de ses attendus. En sa forme pleinement romanesque le réalisme littéraire se donne en effet une pleine liberté de manœuvre dans son usage de « l'illusion référentielle 9 ", encaissant à tous coups les dividendes d'une "représentativité " sociale acquise d'avance par un texte qui peut toujours compter sur la sociologie spontanée du lecteur pour lester ses « effets de réel » : c'est même dans cette croyance du lecteur en la vérité de la fable comme fable que réside la définition de l'effet proprement littéraire, lorsqu'il est obtenu dans le cadre d'une littérature référentielle ${ }^{10}$. Serait-ce alors que, chez Hoggart, les procédés de la description littéraire se verraient au contraire instrumentalisés au service d'une sociologie retorse, d'abord soucieuse de servir les effets de connaissance qu'elle a construits préalablement et qu'elle voudrait imposer au lecteur dans un deuxième temps? Hoggart serait-il un virtuose du marketing littéraire mis au service de l'analyse sociologique? Aurait-il sélectionné par souci tactique les procédures d'écriture 
les plus capables de forcer l'écoute par leur seule vertu suggestive ? S'agirait-il d'un tour de main d'écrivain formé aux bons auteurs - il en cite beaucoup - qui sait la supériorité de la touche légère et du parler droit sur la surcharge ou l'embrouillamini quand il s'agit de ne pas perdre son lecteur en chemin, quitte à effacer derrière lui toute trace de ses techniques de recherche et de preuve?

Ce n'est pas encore cela. Hoggart n'est ni un littérateur flaubertien ni un sociologue masqué. Son registre d'expression est d'emblée celui de la communication littéraire, mais "l'imagination sociologique " (au sens de Wright Mills) est tout aussi directement à l'œuvre dans la construction du monde qui offre la densité de ses faits et de ses événements au romanesque hoggartien : comment un tel romanesque réussirait-il sinon à être si souvent à la fois prosaïque et poétique? Qu'elles portent sur des émotions, des actions ou des catégories mentales, ses analyses documentées, ses observations de longue haleine ont le phrasé de la parole bien écrite mais aussi le nerf de la phrase bien pesée en ses mots et bien pensée en sa méthode. Hoggart écrit comme il réfléchit; il réfléchit en racontant; il raconte en décrivant. C'est un maître de la description argumentée. Mais, disant cela, on n'a pas dénoué le paradoxe : la « description » appartient à la littérature comme aux sciences sociales; simplement, les deux projets descriptifs n'ont ni la même forme expressive ni les mêmes fonctions cognitives. Chez Hoggart l'alliance réussie des deux mouvements de la description, la simultanéité de leurs effets, renforcent leur pouvoir spécifique de communication, au lieu de les annuler ou de les abâtardir par le mélange. À défaut d'en donner la formule, convenons que c'est une réussite assez rare et, pour ce qui nous occupe, une réussite sociologique.

Une ethnographie en filigrane

8 - Mais ceci, c'est la lecture que vous en avez faite. Voulez-vous dire que, si vous n'aviez pas été quelques-uns à avoir remarqué ce livre atypique, si Hoggart n'avait pas été traduit en français et, à l'occasion de cette traduction, «labellisé» comme auteur sociologique, il n'aurait peut-être pas été utilisé comme référence par les sociologues?

- Allez savoir ! Il n'y a jamais d'expérimentation au sens strict en sociologie, et donc pas de vrai «conditionnel passé» dans ce que nous pouvons dire sur un déroulement historique; la sociologie de la réception des textes ne fait pas exception. Mais il est vrai que Richard Hoggart dont l'ensemble de l'œuvre anglaise appartient autant à la critique littéraire qu'à l'étude des "cultures contemporaines" n'est sans doute pas le même auteur pour des lecteurs anglais que pour des lecteurs français qui le lisent d'abord comme un sociologue classique des cultures populaires. Quand, à la fin des années 60 nous avons voulu donner une traduction de The Uses of Literacy aux Éditions de Minuit et que j'y ai mis la main avec deux anglicistes, j'ai peut-être pris conscience que pour faire lire Hoggart en France, il fallait forcer la note, le nationaliser sociologue, en prenant occasion d'une traduction qui tombait à pic dans les débats de l'époque, puisque de toute façon la traduction détruit, ou ne transpose qu'imparfaitement, les effets propres d'un style qui reste toujours solidaire de sa langue naturelle d'origine. Non que la France intellectuelle de l'époque fût avide de franche sociologie, l'étant plutôt d'hyperboles idéologiques sur les mass media ou de débats politiques balisés par les intellectuels de gazettes. Mais justement la véridicité sociologique s'imposait comme la qualité la mieux exportable de ce livre à contre-courant, la force dont il fallait se saisir pour imposer l'écoute d'un ton singulièrement vrai dans la description des cultures populaires; celle, en tout cas, qui pouvait survivre à la traduction, puisque la reconnaissance de l'auteur par 
un public français - toujours plus sensible que d'autres au labelling par le genre - ne pouvait en l'occurrence s'ancrer dans une légitimité littéraire déjà divulguée.

De là l'insistance que j'ai mise, dans la « Présentation » de l'ouvrage, à forcer la main au lecteur, ou plutôt à guider son œil, en lui détaillant les catégories de la description hoggartienne comme une grille ethnographique implicite, comme un raisonnement sociologique en pointillé. De là, surtout, l'effort un peu scolaire investi dans la confection d'un Index articulé et assez détaillé, qui fournissait un moyen de circuler dans l'ouvrage, de le lire complètement, en appliquant au texte les concepts sociologiques ou ethnologiques, les mises en relations signifiantes que la fluidité de l'écriture hoggartienne ou ses understatements argumentatifs allégeaient modestement: ce n'était que justice rendue à un ouvrage d'une grande cohérence théorique, à un inventaire empirique dont l'ampleur panoramique, tricotée très serrée, nous apprend bien plus, sur la vie populaire anglaise, que l'addition de nombreuses monographies consacrées chacune à une tête d'épingle; et davantage, cela va de soi, que les parcours idéologiquement fléchés ou les recensements triviaux de privations déjà répertoriées.

11 Aujourd'hui encore je ne pense pas avoir exagéré. L'explicitation de ce qui s'entend sans prothèse lexicologique a toujours quelque chose de pédant pour l'amateur de finesse. Je craignais la réaction humoristique d'Hoggart à ma «Présentation» chaussée de plomb. C'était lui prêter la condescendance oxfordienne dont, l'ayant lu, j'aurais dû le savoir exempt. Il me répondit naturellement avec toute la "tolérance », dont il avait illustré la portée dans ses descriptions de l'ethos populaire: en laissant traduire mon petit pavé parisien dans un text-book anglais, sous le titre, je crois, de "L'ethnologue en banlieue ». Index ou pas - de toute manière, en France, on ne se sert guère des index - le livre s'est imposé comme un livre de belle et bonne sociologie : c'est qu'il l'était sans le savoir, vous dirait Monsieur Jourdain - anticipant en cela la définition que donne aujourd'hui du «sens» d'une œuvre la sociologie de la «réception» lorsqu'elle introduit en tiers le lecteur entre l'auteur et le texte.

12 Il est facile de faire l'éloge de Hoggart comme ethnographe de la citadinité populaire en Angleterre : sa description de «terrain » est instruite d'une longue immersion au sein des groupes et des espaces dont il parle - c'est la seule définition possible de la méthode ethnologique, dès qu'on ajoute une discipline de pensée à quelqu'esprit de suite dans la recollection des données - personnages, lieux, habitats, parentés, moments rares de bonheur ou litanies du malheur, boulot, sexualité, vêtement, odeurs, spectacles, sports, lectures, religion, superstitions, sentiments, institutions, modèles et nuances de la mentalité ou du langage, de l'esthétique ou de la morale, l'argent du gaspillage comme celui des économies, la défécation comme la nourriture, rien ne manque. Sa biographie même, en l'éloignant sans cesse d'un milieu dont il ne détachait ni ses regards ni ses sentiments, lui a fourni non seulement le matériau mais aussi les angles de vue successifs qui, recomposés avec méthode, font le relief d'une image sociale, lorsque l'observation personnalisée est travaillée par le désir d'objectivité, qui n'est pas autre chose, dans les sciences sociales, que le désir de rendre justice au sens des conduites, de toutes les conduites, des cohérences comme des incohérences, en n'oubliant aucune information. Pour se connaitre psychologiquement sans complaisance ni reniement dans l'ascension sociale qui a fait l'objet le plus constant de sa réflexion rétrospective, Hoggart a été conduit à vouloir connaître obstinément les déterminations sociales liées à son origine populaire et ce qu'elle en a répercuté dans son itinéraire, à la fois linéaire et improbable d'orphelin, de boursier, d'étudiant, de mobilisé, d'universitaire, de chercheur, de 
fonctionnaire international. La garantie de la distance sociologique tient ici au désir de se comprendre subjectivement dans l'objectivité du monde social; de se dépeindre intimement dans ses appartenances. La généralité de la connaissance ainsi produite découle du projet de pensée le plus singulier qui peut venir à un auteur, celui de ne pas se penser comme une exception.

Une sociologie sans tapage

13 - Mais il y a loin d'une ethnographie, surtout implicite, à une théorie sociologique...

14 - Assurément, mais Hoggart " va loin ", sachant ménager sa monture. Par-delà la justesse de la description culturaliste qui s'apprécie au premier coup d'œil, c'est bien la cohérence théorique de ses hypothèses interprétatives qui a très tôt influencé mon travail de sociologue. Les lignes de force de la sociologie hoggartienne sont assurément plus allusives que les traits fermement dessinés de ses portraits ou de ses tableaux, d'autant qu'elles s'expriment le plus souvent dans les incises d'une polémique souriante, dans les digressions d'une interrogation de moraliste ou dans les cheminements d'une question de mémorialiste. Mais derrière l'impression que suscite une notation d'Hoggart, celle de la précision du trait, il y a toujours une piste qui mène loin, en tout cas vers la sociologie, peu pratiquée, de la constitution sociale des affects; sa théorie est discrète, mais elle doit aussi son invisibilité à ce qu'elle s'écarte des sentiers battus, en tout cas des espaces théoriques où s'agglutinent les paparazzi : les théories sociologiques ont aussi leur audimat où les reporters pèsent lourd.

15 Trois exemples au hasard tirés du premier ouvrage. D'abord celui d'une évidence, pourtant éludée ou biaisée par tant de monographies ouvrières: une sociologie conséquente des classes populaires a pour objet principal la vie et la pensée du plus grand nombre, et non cet objet privilégié mais improbable qui fascine les compagnons de route, celui qu'incarnent les personnages «originaux» du militant politique ou syndical, du héros autodidactique, du révolté ou du désespéré solitaire. À prendre ces figures rares, mais familières aux intellectuels, pour la "vérité » de la classe, on a déjà épousé une théorie sociologique de la "conscience de classe», la théorie hégéliano-marxiste qui oblige à définir un collectif par son « maximum de conscience possible », ce maximum fûtil intermittent, minoritaire ou seulement lisible aux philosophes de cabinet. Sans même avoir à invoquer les certitudes plombées de la théorie, la sensibilité des écrivains révolutionnaires ou anarchistes et, plus largement chez la plupart des écrivains, la recherche d'un maximum de relief pittoresque suffisent à focaliser le traitement romanesque du peuple sur les seuls membres du groupe qui s'en détachent du fait même qu'ils expriment individuellement ou minoritairement une de ses passions fortes : c'est là la définition du "héros populaire» en toute littérature qui se veut bienveillante au " peuple »; dans la littérature anglaise c'est aux personnages de Jude l'obscur ou de Félix Holt que se réfère Hoggart pour caractériser ce populisme littéraire, cette mise en littérature aussi chaleureuse que trompeuse ${ }^{11}$.

16 Mais on s'interdit alors de décrire une des contraintes sociales les plus déterminantes de la condition générale des classes populaires à savoir: "l'absence de perspectives » individuelles, la grisaille d'un destin collectif anticipé comme certain d'avance et - du moins jusqu'à la naissance récente des espoirs vite déçus de voir tous les enfants de la famille échapper à la classe sociale par la scolarisation - la banalité d'un destin destiné à se répercuter monotonement d'une génération à l'autre. Ignorant les effets les plus constamment associés à la condition de masses pauvres et dominées, on perd ainsi l'occasion de ressaisir une des productions symboliques les plus caractéristiques de la 
condition populaire, cet univers de sentiments, de gestes et d'idées, ce "cosmos qui est doté de sens ${ }^{12}$ » par l'effort déployé quotidiennement pour maîtriser psychologiquement un environnement d'adversités et de contraintes. C'est cet univers culturel, instrument symbolique d'une maîtrise gardée " en dépit de tout ", par les moyens du bord, que décrit minutieusement Hoggart, ce monde collectif d'échanges intenses et de valeurs communes, de conflits et de solidarités, ces plaisanteries faites sans grande joie, ces espérances sans grand espoir, cette gaieté ou cette « débrouillardise » de nécessité, cette vivabilité mentale aménagée au sein même d'une condition à première vue invivable, cette énergie faite des mille et une manières de "faire avec ", cette dénégation à la fois énergique et désabusée de l'anonymat et du malheur sans éclat qu'un avenir bouché, raccourci, immobile impose aux biographies écrasées et si tôt jouées des gens du peuple - entendons ceux qui en sont parce qu'ils y restent. Bref, on perd ce qu'il y a de plus culturel dans la culture populaire comme "culture du pauvre " pour n'en retenir que ce qui en est le plus idéologique comme idéologie de la lutte, de la revanche, de la résignation ou du désespoir. Non qu'il n'y ait pas d'idéologie (au sens d'une mise en discours suivi des affects de position) dans la culture où vit et se pense la majorité des membres des classes populaires, mais il y en a moins, et elle est toujours moins unifiée et plus sporadique que dans une culture savante ou une culture de privilégiés; en tout cas moins grandiloquente que dans une culture intellectuelle, et surtout que dans celle des intellectuels populistes qui «vont au peuple " comme à une voie de salut, partageant tous quelque chose de l'irréalisme lyrique qui fut celui des narodniki russes ${ }^{13}$ - tous, même, quand ils en sont issus, ceux qui "y reviennent», parce que le peuple qu'ils retrouvent ne peut plus être, comme objet de pensée, le même peuple que celui dont ils sont sortis.

Autre exemple: il suffit de quelques lignes à Hoggart pour déstabiliser par une observation les théories des cultures «nationales ", "régionales » ou micro-locales, qui invitent presque toujours à substantialiser les identités culturelles comme autant de monades juxtaposées partes extra partes dans l'espace géographique ; à les penser comme autant de petits mondes refermés sur et dans leur autonomie signifiante; à les supposer descriptibles comme autant de spécimens ou de cailloux - de patterns of civilization disait Ruth Benedict. Hoggart nous fait remarquer à partir d'un souvenir personnel (il a été mobilisé pendant la deuxième guerre mondiale) que des ouvriers anglais sous l'uniforme invités dans une famille populaire napolitaine y étaient, malgré la barrière linguistique, aussi immédiatement à l'aise dans la gaité conviviale autour d'un plat de pâtes vernaculaires que dans les échanges chaleureux de leur home et du voisinage ou autour des bières euphoriques d'un pub anglais. Portés par leur spécialisation sur une aire à la géographisation de leur typologie et, par là, à la sur-estimation "culturaliste ", les anthropologues n'exagèrent-ils pas la distance entre une culture aussi ostensiblement exubérante que la napolitaine et celle de ces petits soldats venus du brouillard et de la misère lointaine des banlieues anglaises? Hoggart nous suggère ici, à contre-pente du "culturalisme", qu'un ouvrier anglais pourrait bien être, en ses interactions sentimentales, en sa gestuelle et ses communications non verbales, plus proche du petit peuple d'un pays méditerranéen qu'il ne l'est des classes moyennes de sa propre société ? L'internationalisme ouvrier ne s'est jamais avisé que de tels constats opérés au plus près de «l'interaction symbolique » étaient bien mieux ajustés à la réalité des distances et des proximités entre classes ou groupes que ses trop vagues argumentations théoriques plaidant laborieusement, aux fins de propagande, l'identité des intérêts économiques de tous les dominés par-delà les frontières nationales. 

apparu dans les années 60 comme un fleuve sociologique tranquille qui, fort de sa familiarité, vérifiée et revérifiée, avec les mœurs populaires d'une grande cité du début du siècle, évitait comme en se jouant toutes les erreurs alors à la mode sur le rapport mystifié et crédule des "masses » aux mass media, sur le rapport du peuple à la culture savante - qui n'est ni de honte culturelle ni de prosternation - sur la « résignation » des plus démunis - qui n'est ni une torpeur ni une auto-dévaluation - ou encore sur le «cynisme " populaire, la «débrouillardise», la «vie au jour le jour»-qui ne sont ni pensée à courte vue ni ce qu'en pensent cursivement «les autres». Bref Hoggart me révélait, par la cohérence construite d'une série de traits culturels, d'autres formes de la rationalité pratique que celles, bourgeoises ou petites bourgeoises, qui monopolisent égocentriquement, chez les théoriciens de l'économie comme chez les maîtresses de maison conformistes, la définition de toute " rationalité ». Sans avoir besoin de recourir à la polémique que Bourdieu et moi-même mobilisions alors défensivement pour dénoncer la vanité scientifique de la "mass-médiologie » (qui attendait encore son théoricien le plus excessif avec Mc Luhan) ${ }^{14}$, Hoggart ne tombait dans aucun des panneaux où, en cette période, essayistes et même sociologues patentés s'engouffraient, avec délectation ou cris d'horreur, en décrivant comme somnambules en chambre jamais tentés par une sortie sur le terrain, la " passivité » des masses hypnotisées par l'irruption planétaire des images et «l'aliénation» des cultures populaires chambardées par les nouveaux moyens de communication.

C'est au contraire à partir d'une connaissance instruite de l'ethos populaire que se développe la théorie hoggartienne la mieux développée dans The Uses of Literacy. Son analyse nous livre en clair le modèle descriptif d'une forme paradoxale de "réception ", celle que les groupes dominés appliquent pour leur utilité propre et par nécessité de position, aux messages venus d'en haut et d'ailleurs. Hoggart nous permet de comprendre une attitude populaire, à la fois simple et retorse, spontanée et matoise, qui est régulièrement méconnue par les observateurs pressés ou condescendants: sous l'apparence d'une disponibilité bon enfant, accueillante au sensationalisme de la presse populaire, ouverte sans y regarder de près à la consommation des infra-littératures et des images diffusées en série, prêtant une attention souriante et sceptique aux propagandes politiques, c'est en réalité une attitude qui consiste, à " savoir en prendre et en laisser ", une forme de réception qui trouve dans un acquiescement peu engagé à l'écoute le moyen de «ne pas s'en laisser conter» par le message, attitude de défense, peut-être plus efficace que la polémique intellectuelle ou l'indignation morale; attitude paradoxale en tout cas dont j'ai essayé de rendre la subtilité par l'expression d'«attention oblique » ou « distraite».

Ce « faire avec » révèle bien un calcul qui, pour être souvent bouclé d'avance dans une sagesse collective, n'en relève pas moins d'une rationalité adaptée à la pauvreté des moyens d'influence et de contrôle qui est caractéristique d'une condition dominée : il s'agit bien là d'une rationalité spécifique capable de retourner le maximum de handicaps en atouts, ou au moins en ces petites ruses journalières qui permettent de prendre la tangente, là où l'on ne saurait faire face dignement ou victorieusement. La sagesse populaire que décrit Hoggart a quelque chose de «stratégique » : éviter au maximum les terrains où la confrontation avec « les autres » serait désavantageuse. Les gens du peuple ne sont pas des « polyhandicapés » culturels comme le ressassent à l'envie la sociologie et la littérature misérabilistes; ni des enfants ou de «grands enfants », enfermés dans le 
jardin enchanté de la magie ou la naïveté du « premier degré », comme le susurrent avec le plaisir du "second degré " les intellectuels populistes, qu'ils soient littérateurs, cinéastes ou sociologues. Je ne sais si la théorie hoggartienne de la spécificité des ressources que les gens du peuple tirent du dénuement de leur condition et de l'infériorité de leur position est une théorie sociologique ou psychologique (cela ne concerne que le style d'intelligibilité qu'on lui prête). Mais je sais, si je me laisse guider par l'enquête, que toutes les théories qui ignorent cette philosophie et cet art propres aux classes populaires de protéger un quant-à-soi psychologique et de gérer ainsi un incessant va-et-vient entre l'autonomie et l'hétéronomie symboliques de leurs représentations, reposent sur une paresse descriptive qui ne persuade l'incuriosité des lecteurs cultivés que parce que l'increvable notion d'« aliénation » a acquis le statut scolaire d'une vulgate.

L'autobiographie hoggartienne comme art du romanesque sociologique

21 - On aperçoit encore mieux l'art, propre à Hoggart, de construire une connaissance sociologique d'autant plus persuasive qu'elle est mieux tissée des fils d'une émotion et d'une mémoire personnelles dans son dernier ouvrage, A Local Habitation publié en $1988^{15}$, qui vient d'être traduit au Seuil. Comme on le voit à la «Présentation» qu'en donne Claude Grignon, ce deuxième livre pose à un autre sociologue le même problème que me posait le premier, celui d'élucider la réussite improbable d'un mariage entre l'eau et le feu, la froideur d'une analyse des faits qui enseigne au lecteur la distance scientifique et la vibration immédiate d'une écriture et d'une composition qui induisent la proximité littéraire. Si The Uses of Literacy était déjà - Hoggart ne s'en cachait pas - la mise en chapitres d'une documentation biographique, le passage déclaré à l'autobiographie ("Autobiographie d'un intellectuel issu des classes populaires anglaises » dit le sous-titre choisi par les traducteurs français) est ici constitutif d'un projet qui entremêle encore plus savamment les éléments que l'auteur s'était naguère obligé à ventiler dans des rubriques séparées. Hoggart est passé de la biographie per species (à la manière de Suétone) à la narration per tempora (à la manière de Tacite) ou, si l'on préfère, des fiches de l'ethnographe ou du géographe au récit argumenté et contextualisé, là où se rencontrent et se mesurent l'historien qui croit aux vertus explicatives d'un ordre de la "diégèse » et le romancier qui confie ses chances d'être lu aux effets littéraires du romanesque « référentiel ».

Le passage à l'autobiographie se présente en effet dans l'œuvre de Hoggart comme l'entrée dans un espace de l'écriture où s'accroissent à la fois les libertés d'allure du récit et les contraintes de la preuve ou de la présomption ${ }^{16}$. Ce faisant, il ne passe pas de la sociologie à la littérature comme d'un camp au camp opposé : la liberté d'interprétation que l'auteur s'accorde dans le travail de mémoire pour organiser ses reconstructions signifiantes comme autant d'interprétations du devenir individuel ou collectif ouvre au chercheur de nouvelles possibilités de mises en relations; elle oblige, me semble-t-il, à encore plus de justesse le style sociologique qu'adopte Hoggart pour décrire ou narrer trajets, figures et situations. Plus la théorie sociologique qui associe dans un ordre explicatif la volonté de culture et le destin social du narrateur se trouve gommée en ses formulations les plus abstraites, plus forte apparaît l'adéquation de cette théorie à la forme d'écriture choisie par l'auteur.

23 Mais faut-il dire "gommer» pour désigner cet effacement de l'ordre théorique du discours qui préserve pourtant, en même temps que la lisibilité sociologique, l'accès d'un large public à l'argumentation explicative de l'auteur comme au sens reconstruit par le 
narrateur ? Un tel « gommage » ne ressemble absolument pas à celui qui, dans les arts les plus savants, est stylistiquement calculé au millimètre pour aguicher un public de " connaisseurs ", friands de se trouver reconnus comme public légitime par une œuvre qui leur réserve le plaisir de réussir des reconnaissances difficiles et rares. L'estompage de l'«argument " n'est pas ici une invite latente adressée aux happy few sociologues, comme le gommage du «sujet" peut l'être, en direction d'autres élites, dans un art volontairement énigmatique destiné à des commanditaires subtils, celui par exemple que Salvatore Settis décrit comme constitutif de la manière picturale de Giorgione, gommant savamment de reprises en reprises les iconographies trop reconnaissables de La Tempête ou des Trois philosophes ${ }^{17}$ ?

Hoggart n'a pas eu à "gommer » le langage d'une sociographie ou d'une ethnographie préalables. Organisé par son projet personnel de faire comprendre son propre itinéraire social, libéré des rubriques et des marqueurs de la recherche ostensiblement universitaire, son regard sociologique est d'emblée modeste, dilué dans le matériau romanesque, conceptuellement discret, mais le filigrane de l'intelligibilité sociologique court toujours comme une forme noyée dans la matière. En somme, en ce nouvel ouvrage, Hoggart fait plus et mieux que jamais dans la subtile manière Hoggart. Explication et compréhension, "adéquation causale » et " adéquation significative ${ }^{18}$ » sont ici étroitement associées, comme en tout discours qui produit de la connaissance sociologique, mais par un cheminement qui ne laisse apercevoir qu'à l'arrière-plan le panorama organisateur des typologies sociologiques. Tout l'art d'Hoggart, que l'on appellera, si l'on veut, celui du romanesque sociologique, en le distinguant ainsi du romanesque social, tient à ce que l'arrière-plan théorique imprègne les détails les plus concrets du premier plan: la sociologie hoggartienne suppose un rapport de la « figure » au « fond » qui ne se laisse évidemment pas définir par une formule ou un formalisme; mais la teneur sociologique du récit hoggartien se prête, sans qu'on puisse hésiter, à la reconnaissance d'une «forme » qui peut se « désigner » nominalement, comme après tout on se résigne à le faire pour une « marque » artisanale ou artistique.

Dès la première phrase du premier chapitre, qui a frappé plus d'un critique et qui fait entrer le lecteur dans le pacte littéraire d'une narration autobiographique et d'une émotion en première personne - « Ma tante Annie est en train de mourir à l'Hôpital Saint James » - on voit à l'œuvre toute l'efficacité combinatoire du mouvement propre au récit hoggartien, puisque cette phrase est indissociable, pour qui veut l'entendre pleinement, du paragraphe qui suit où l'auteur confie à son lecteur la nuance expressive qui démarque l'emploi du pronom possessif de celui de toute autre marque dans la parlure populaire du Nord-Est de l'Angleterre. Sans l'art hoggartien du fondu-enchainé, la juxtaposition d'un incipit littéraire et d'une remarque de socio-linguistique ne serait qu'une recette. Cet incipit ne se compare donc pas au "C'était à Mégara, faubourg de Carthage » ou au «Longtemps je me suis couché de bonne heure » de noble mémoire. Ici, la connaissance analytique d'un minuscule trait culturel se révèle indispensable, dès lors qu'elle est délivrée amicalement au lecteur, pour exprimer pleinement un sentiment personnel ; et, simultanément, l'écriture directe de l'émotion est seule à pouvoir transmettre tout ce que signifie localement et subjectivement le langage social de la sensibilité familiale, puisque les fils ainsi noués entre l'objectivité du contexte et la subjectivité des représentations vont être tissés de plus en plus serré tout au long du livre: l'analyse du sens social des conduites et des mots de la vie quotidienne fait le retentissement littéraire des épisodes 
de la mémoire; mais ce mouvement n'est pas distinct de celui qui permet au retour autobiographique de construire la connaissance sociologique en l'exemplifiant.

Il est facile de constater en suivant au long des chapitres les sinuosités du récit hoggartien que la psychologie du narrateur procure, dans les diverses phases de cette histoire de "formation", la plus grande partie de leur sens, non seulement aux événements rapportés mais aussi à l'intelligibilité sociologique qui découle de leur analyse par l'auteur. Mais, chez le lecteur accoutumé à la lecture des «romans de formation" s'insinue vite une impression paradoxale de trans-psychologie, ou, plus précisément, la sensation originale que le récit hoggartien repose sur un déplacement méthodique du sens des actions humaines, sens qu'il s'est depuis longtemps habitué, en tant que lecteur de "romans psychologiques", à identifier comme une intelligibilité intemporelle de l'intrigue reposant sur la continuité et l'autosuffisance psychiques des personnages. À quoi tient cette impression de renouvellement par Hoggart du ton de l'autobiographie ? On aperçoit évidemment d'emblée qu'il n'existe pas dans le récit de vie que nous propose Hoggart une seule nuance du sentiment ou de ses transformations qui ne soit restituée par la description ou l'argumentation de l'auteur aux conditions et aux interactions sociales dont elle est solidaire: mais cela ne suffit pas à caractériser spécifiquement le romanesque hoggartien puisque cette intelligibilité qu'on appellera psychosociologique irrigue aussi chez bien d'autres auteurs les impressions de " comprendre " en toutes sortes de genres et de veines littéraires. Chez Hoggart le « social»- le contexte, l'ancrage social des sentiments et des consécutions d'actes - est beaucoup plus constitutivement et activement romanesque : il n'est pas réduit, comme en de nombreux genres littéraires, à jouer le rôle décoratif d'une couleur locale du "psychique", d'un habillement spatio-temporel particularisant une histoire dont la signification humaine pourrait encore être racontée comme telle dans sa nudité psychologique, y perdant certes l'attrait de ses accidents pittoresques, mais en définitive rien de son essence signifiante. C'est le refus, modeste mais circonstancié, d'entrer dans une telle philosophie de la connaissance du «cœur humain» - de l'« âme humaine » comme disaient les professeurs de lettres classiques commentant au registre du grandiose la constance des grandes passions de l'homme, à travers la diversité de l'histoire ou des milieux sociaux - qui fait de notre auteur un sociologue ${ }^{19}$; et de son romanesque - où la description de l'espace social d'un sentiment est constitutive de la communication au lecteur de son sens vécu - un romanesque sociologique.

Parmi toutes les petites évocations qui font dans leur diversité l'unité de ton - sociologique, littéraire et affective - de la remémoration hoggartienne, retenons, par exemple, la description en écheveau de toutes les saveurs qui adhèrent au Spartiate " gâteau de four » de sa Grand-Mère, puisque ce souvenir conduit l'auteur à placer son propre style d'évocation des moments de "bonheur inattendu et sans mélange " sous le signe de son admiration pour Tchekhov « entre autres raisons pour son acharnement à décrire de tels moments, comme dans la nouvelle Après le théâtre où il parvient à rendre le sentiment du contentement à l'état pur, dépourvu d'affectation, chez une jeune fille ${ }^{20}$ ". La recette minutieusement décrite de ce gâteau de ménagère économe ainsi que les sensations du plaisir gustatif pris à cette cuisine du pauvre réussissent bien à transmettre au lecteur tout le retentissement de « ce qui a été expérimenté ce jour-là sur le seuil de la maison, davantage que le plaisir élémentaire qu'éprouve un garçon à manger un en-cas succulent »; Hoggart nous fait ressentir en deux paragraphes "la sensation fugitive d'être complètement en accord avec le monde, d'être en paix»; mais il faut 
qu'interviennent aussi, outre une réflexion d'Hoggart écrivain de langue anglaise sur ce qu'est l'écriture en demi-teinte, le personnage tutélaire de la Grand-Mère, la belle saison, le week-end à domicile, la réussite du boursier à l'École et la suspension de sa discipline hiérarchique, la vie difficile et le répit, bref toute la condition sociale qui fait l'objet du livre, pour que la «sensation d'être simplement une parcelle apaisée de l'univers, le sentiment anxieux et sans fin de l'inachevé suspendu pour un temps » prennent tous leur sens, celui d'une expérience intense de la réconciliation avec soi, dont Hoggart ne voit dans sa vie que trois autres exemples, à part le sentiment amoureux, et le dernier « au chevet de Tante Annie ». Est-ce le «sens » psychologique ou le «sens » sociologique qui anime ici l'espace de la "compréhension» d'une biographie, ou qui fait le nerf et la couleur d'un romanesque? La manière hoggartienne pourrait se définir par l'art de rendre difficile la réponse à cette question. Mais il faut lire le livre puisqu'il ne se résume pas : disant cela, on voit que je conseille la lecture littéraire d'une œuvre sociologique - mais on pourrait aussi bien dire la même chose en inversant la formule - puisque la valeur sociologique de l'information et de l'argumentation y est inséparable d'un art de l'écriture et de son pouvoir de suggérer.

En quoi l'œuvre de Hoggart

contribue-t-elle à une sociologie des intellectuels ?

- In cauda caput! Prenons acte que le temps nous manque pour l'essentiel. Vous me posez la question la plus importante, puisqu'elle conduit directement à l'originalité sociologique de l'autobiographie d'Hoggart, au point précis où la galerie des portraits intellectuels et des paysages institutionnels attendus tout au long d'un tel parcours nous est donnée à voir sous un angle inattendu, celui de la valeur objective d'un tableau dont le regard subjectif du narrateur est le garant paradoxal par la singularité même de son orientation sociologique. Hoggart se révèle ici comme un intellectuel issu des classes populaires plus libéré et pourtant plus proche de son origine de classe, moins crispé ou désinvolte, moins embarrassé ou glorieux, et pourtant plus attentif à tout ce qui découle de cette origine, plus atypique donc en son parti de remémoration que presque tous ceux qui ont pris la plume pour se raconter, y compris les mémorialistes dont le parcours social et intellectuel pourrait sembler le plus comparable au sien : je pense par exemple à Jean Guehenno ou Louis Guilloux en France - pour ne pas s'éloigner trop du cas des enseignants venus du peuple, en étendant la comparaison aux Confessions d'un écrivain glorieux comme Jean-Jacques, d'un autodidacte à l'itinéraire picaresque comme Georges Navel ou d'un philosophe politique aux appartenances enchevêtrées comme Althusser.

La contribution de Hoggart à la sociologie des intellectuels est double. Je mentionnerai d'abord la richesse et la diversité typologique des portraits psychologiques et sociaux d'intellectuels issus des classes populaires : « boursiers » décidés à la réussite ou résignés à la demi-réussite, ratés pathétiques voués à la névrose d'échec vécue comme une guigne, parvenus qui « en remettent » un rien de trop dans une virtuosité où se trahit l'emprunt, hommes habités par le ressentiment ressassé ou la capitulation amère, lettrés ou savants installés dans l'establishment au prix d'un oubli de leur origine, assez proches finalement, par la véhémence de leur dénégation silencieuse, des self-made-men ou des diplômés hâbleurs qui cultivent l'autoglorification par la magnification des vertus intellectuelles et morales prêtée à une ascension miraculeuse opérée à partir du néant social. Hoggart luimême, c'est-à-dire l'homme que révèle un tel style autobiographique ne peut se lire, on le voit, selon aucune des grilles biographiques qui sont exemplifiées dans le livre. Non qu'il prétende planer dans un ailleurs absolu réservé à un auteur « sans racines » ou être resté 
plus fidèle, par sa franchise, à l'éthique et aux affects de son milieu d'origine que ceux qu'il a côtoyés dans son ascension sociale; mais l'autoportrait de l'auteur est trop riche pour ne pas rester ambigu puisqu'il est démultiplié par les fonctions de l'écriture, se réfractant différemment dans les "moi » successifs du narrateur et dans les tâches entremêlées que s'est données un écrivain voué à la fois à l'anamnèse psychique et à l'étude sociale. Le portrait de Hoggart considéré comme un personnage de son propre récit est nécessairement celui d'un "héros problématique " puisque, informateur et interprète, narrateur et auteur, collecteur de mythes et démystificateur, il est le sujet et l'objet de tout le livre qui, une fois refermé, laissera le lecteur - sociologue, moraliste, amateur d'âmes ou de cas - seul juge, perplexe ou conquis, de la sincérité psychologique du récit comme des vertus sociologiques de l'analyse. «L'œuvre de chaque homme est toujours un portrait de lui-même » dit Hoggart citant Samuel Butler.

N’oublions évidemment pas, dans la biographie d'Hoggart, la présence des médiateurs et des personnages intellectuels venus d'ailleurs que des bancs de la lente démocratisation de l'École : l'itinéraire du narrateur le conduit en effet de sa rencontre avec la culture scolaire et des premières curiosités pour les livres lus à la bibliothèque municipale d'Hunslet au contact rugueux des agents tutélaires de toute ascension par l'École. Au passage, portrait d'une assistante sociale d'un autre âge, une Miss Jubb, «visiteuse du Comité des Gardiens", qui parle comme dans Dickens et dont Hoggart compare avec amusement le moralisme autoritaire à la pratique subversive des "travailleurs sociaux " qu'il croise aujourd'hui dans des stages, ramassant humoristiquement dans ce raccourci de deux pages l'inversion idéologique qui a fait passer d'une idéologie du "garde-chasse » à une idéologie de «braconnier ${ }^{21}$ » les agents subalternes de cet appareil d'encadrement de la misère - joli survol historique qui dément avec la légèreté du conte la pesante théorie de la pérennité des fonctions de domination prêtées aux « appareils idéologiques d'état » par les philosophes amis du peuple. Portraits aussi de pédagogues où la diversité des caractères est indissociable des nuances du statut social ${ }^{22}$ et, parmi eux, celui d'un directeur d'école primaire anglaise aussi attaché à l'ascension scolaire de ses élèves méritants et prometteurs qu'un « instit » français du début du siècle, mais dont le goût du « simulacre » vestimentaire et la propension à se donner une allure de classe supérieure à la sienne - petitesses conformistes d'un petit-bourgeois anglais - déconcerteront ceux qui sont enclins à voir dans l'idéalisme militant des « hussards de la République » le modèle sans patrie du dévouement pédagogique aux enfants du peuple.

31 La typologie se diversifie encore lorsqu'on passe aux portraits des professeurs et du proviseur du lycée de Cockburn, gate-keep-ers de l'entrée dans la culture scolaire et son langage châtiéé ${ }^{3}$; ou encore, de l'autre côté de la frontière de classe culturellement et linguistiquement si abrupte en Grande-Bretagne, lorsque le narrateur découvre l'étrangeté de l'establishment et du « beau monde ", l'exotisme de ses femmes par exemple, " minces et jolies, avec un accent que (le narrateur) identifierait sans doute maintenant comme un accent de public school [...], qui nous ignoraient totalement (les élèves d'un quartier populaire en virée, qu'elles gratifiaient d'un pourboire), pour qui nous étions comme des coolies pour des sahibs ${ }^{24} »$ : le lycéen se souvient « surtout de leur odeur [...], une odeur de parfums qui suggéraient quelque chose d'exotique et de remarquablement cher. Ce n'était pas une odeur sexy; c'était une odeur d'insouciance, d'allant, d'aisance financière. Il y avait donc un monde dans lequel des jeunes femmes sveltes, élevées dans des parties de l'Angleterre dont nous avions seulement entendu parler, avec des noms tels que Egham, Midhurst, Haslemere et Sevenoaks se déplaçaient librement et faisaient 
ce dont elles avaient envie... etc. ${ }^{25}$ ». Enfin, last but not least, la culture lettrée s'incarne dans les portraits de quelques universitaires, auteurs ou étudiants de type oxbridgien ${ }^{26}$ - bien que l'Université soit celle de Leeds - eux-mêmes plus vivants et divers en leurs biographies et leurs physionomies qu'en leur stéréotype reçu sur le continent. Les passages consacrés à Bonamy Dobrée qui assume, dans l'étape décisive de la formation de l'auteur, le rôle du " père intellectuel », prenant ainsi la suite du Directeur d'école de Jack Lane et du Proviseur de Cockburn, ne sont pas le simple hommage d'une reconnaissance émue; on y trouve l'esquisse d'une sociologie subtile des rapports interactifs entre un disciple et son maître, et par exemple cet aiguillage par le maitre de la curiosité du disciple vers un "ancêtre " de prédilection, l'écrivain américain du XIXe siècle Henry Adams, dont «Dobrée pensait qu'il (Hoggart) retirerait de ce livre quelque chose qui ferait fonctionner une nouvelle partie de (son) cerveau ${ }^{27} »$. Perspicacité du maitre, médiateur d'un autre maitre, puisqu'Hoggart nous dit avoir trouvé dans ce "livre étrange » (My éducation) l'exemple d'une « prise de distance vis-à-vis de soi-même » en des termes qui évoquent assez précisément l'intrication du psychologique et du social que nous essayons difficultueusement de formuler à propos de l'entreprise littéraire d'Hoggart lui-même : «Sa confrontation avec les problèmes de forme que pose ce genre d'écrit - comment maîtriser l'interaction de l'expérience personnelle et de la signification collective $^{28}$ - m'aidait dans ma propre recherche, tout à fait similaire, en renforçant le sentiment que celle-ci valait la peine d'être entreprise ${ }^{29}$."

Ce sont les interactions saisies sur le vif entre tous ces personnages, sans oublier le narrateur en ses rôles successifs, et les rapports psychologiques de toutes ces figures aux institutions et aux normes d'un ordre social, illustrant une forme toute britannique des distances hiérarchiques, qui constituent sans doute l'apport sociologique le plus original du dernier livre de Hoggart lorsqu'on le compare au précédent, où la limitation du champ de vision à la famille populaire et à son voisinage de quartier ne laissait transparaître du monde des «autres » que ses hauteurs indistinctes ou ses émissaires locaux, et comme profils d'apprentis intellectuels que ceux du boursier et de l'autodidacte.

Enfin et surtout, je trouve dans A Local Habitation une extraordinaire contribution a contrario, ou plutôt par ricochet, à la question du populisme. En décrivant les formes générales ou localisées de la culture populaire en termes pesés, avec une justesse de ton dans le « rendu » de l'expérience vécue et une patience méthodique dans la récollection des faits qui imposent la véridicité descriptive de l'une comme de l'autre, Hoggart pose implicitement au lecteur, et parfois explicitement, une question insistante : pourquoi la plupart des descriptions sociologiques ou littéraires mettant en scène le «peuple » que nous avons lues ailleurs nous donnent-elles l'impression de nous introduire dans un univers si totalement étranger à celui que décrit Hoggart - et, pour tout dire, dans un univers de «faiseurs »- que ce soit dans celui des bilans dévitalisés d'une sociologie quantitative des privations, dans celui d'une contre-culture unifiée par la lutte ou l'idéologie de classe, ou encore dans celui, au moins aussi trompeur, de la «franche gaieté » et de la "bonne santé », univers présenté par les sociologues ou les romanciers journalistes comme si « authentique » qu'il semble que chacun ne devrait rien avoir de plus pressé que de se précipiter pour aller y vivre? À quoi jouent les intellectuels - de toutes origines et de toutes tendances - quand ils jouent avec l'idée ou le fantasme du " peuple » comme avec une poupée de son ou un chien de paille?

Misérabilisme et populisme 
34 - Est-ce que la découverte, ancienne chez vous, de Hoggart ne vous a pas aidé à ouvrir le débat que vous développez, en dialoguant avec Grignon, dans Le savant et le populaire ${ }^{30}$ concernant l'« oscillation permanente", dans les travaux sociologiques consacrés aux cultures populaires, entre " populisme » et « légitimisme»?

35 - Bien sûr. C'est la lecture de Hoggart qui a alerté ma première curiosité sur ce problème à la fois théorique et méthodologique, aux retombées multiples, sans oublier ses échos politiques. Hoggart a navigué, les contournant d'un évitement intuitif, entre les deux écueils majeurs de la mésinterprétation des cultures populaires, désignant ainsi d'un geste discret les deux repères polaires de l'ethnocentrisme de classe. Le premier repère, qui se résume dans l'hypothèse, intenable jusqu'au bout d'une description, de l'auto-suffisance symbolique des cultures dominées désigne le danger "populiste»; le second, qui se résume dans l'hypothèse, tout aussi intenable, de l'efficacité universelle d'un ordre légitime balise la tentation " misérabiliste ${ }^{31}$.

36 En lisant des enquêtes sur les classes populaires ou en revenant sur le traitement que leur réserve la littérature réaliste du siècle dernier, en écoutant, plus simplement, les conversations petites ou grandes-bourgeoises, intellectuelles ou semi-intellectuelles sur le "peuple ", comme en observant dans l'enquête les membres des classes populaires parler d'eux-mêmes et des "autres ", j'ai progressivement été convaincu que les deux injustices à la fois descriptives et interprétatives, celle du populisme et celle du misérabilisme, étaient parfaitement symétriques, y compris en ce qu'elles procédaient l'une comme l'autre du parti, tantôt naïf tantôt véhément, de rendre, dans la description, justice à la condition populaire.

37 Toute description d'une culture qui entend tant soit peu s'arracher à l'« ethnocentrisme » commence par le choix du «relativisme culturel», charte professionnelle de toute anthropologie, en ce sens qu'il faut commencer par se donner les moyens de décrire les significations qui font l'identité culturelle d'un groupe avant de vouloir les hiérarchiser. Mais, s'en tenir là, c'est enfermer la description dans l'espace clos et idéologiquement trompeur du populisme. Quand les différences culturelles se trouvent hiérarchisées par et dans des interactions réelles - comme au sein d'une même société, entre membres de classes ou groupes sociaux inégaux en pouvoirs et en avoirs - le relativisme intégral, qui se borne à répéter que toute hiérarchie des valeurs est culturellement arbitraire, conduit vite à oublier les effets propres de tout ordre social, c'est-à-dire la «violence symbolique » qui est capable d'imposer la reconnaissance de n'importe quel ordre établi comme ordre culturel "légitime». La théorie de la "légitimité culturelle", venue de Weber et indirectement de Marx, s'impose alors comme un rappel au réalisme sociologique. Refuser ce réalisme dans la description des réalités hiérarchiques, c'est glisser du relativisme de méthode à l'autonomisation, lyrique ou politique, de la culture décrite, et de là, bien vite, au populisme, description idyllique et donc injuste de toute condition dominée, par l'oubli de la domination sociale qui est inscrit au cœur même de ce projet de description $^{32}$.

38 À l'opposé de la complaisance populiste, la stratégie descriptive qui consiste à traquer en toute conduite populaire les marques de la soumission, à tout déduire des effets hétéronomiques d'une domination subie, rendrait-elle pleinement justice aux classes dominées? Évidemment pas. Faites le trajet qui va du constat factuel des légitimités sociales, c'est-à-dire des effets objectifs de tout ordre légitime, à la thèse qui postule mécaniquement la reconnaissance subjective de la légitimité par ceux que cette reconnaissance plonge illico dans l'illégitimité, et vous verrez aussitôt comment, dans les 
mots dits ou sous-entendus du discours sociologique, on glisse de la théorie de la légitimité culturelle au "légitimisme» (en un sens fort comparable à celui que lui a donné le lexique politique). On oublie ce faisant toutes les significations culturelles qui, dans une culture populaire, ne peuvent s'énoncer, sous peine de s'évanouir, qu'en termes d'autonomie symbolique; on perd de vue les comportements et les représentations qui échappent à l'obsession de la légitimité ; on ne voit plus dans une culture populaire que dépendance mécanique ou dénégation de la dépendance, ce qui revient, dans les deux cas, à se donner l'essence du peuple comme hétéronomie de comportement, comme « conscience aliénée » de soi : Hegel ou Feuerbach - qu'on les invoque ou non - sont les seuls garants de cette certitude chère au philosophe de détenir la « conscience vraie » (de l'histoire, de l'homme ou du peuple). C'est par cet oubli de l'autonomie irréductible du symbolisme dominé que le sociologue est conduit tout droit au "misérabilisme", comptabilité apitoyée des absences, des privations et des manques à quoi se réduit une culture populaire dès qu'on n'y voit plus qu'une culture dominée, définie tout entière par les effets de la domination. Le misérabilisme a pu revêtir mille formes depuis les plus hautaines jusqu'aux plus charitables, mais la déploration ou la compassion ramènent toujours ses chiffres implacables ou ses bons sentiments au mépris souterrain. La machine mentale qui, à propos du «brave» ou du "pauvre peuple», enchaîne si imparablement les omissions et les bévues, les mépris et les paternalismes, est de surcroît une machine à double détente qui réussit fort bien à additionner, en nombre d'analyses plus soucieuses du pathos que du concept, les cécités contradictoires du misérabilisme et celles du populisme.

Cette machinerie, bien plus efficace qu'une machination, obéit d'ailleurs à la même mécanique en sociologie et en politique, même si les dégâts n'y ont pas la même forme, ici de description mutilée, là de monoïdéisme idéologique. La machine tourne encore plus rond en littérature, puisqu'elle est là sollicitée de "singulariser » le peuple en procédant à l'exotisation de l'altérité intime d'une société, ressort toujours facile et efficace d'une "désautomatisation» bienvenue au plus grand nombre de lecteurs ${ }^{33}$. Mais il serait interminable d'énumérer les biais et les effets pittoresquement ou naïvement déformants du populisme ou du misérabilisme dans les descriptions littéraires : Hoggart en parle fort bien à propos de la littérature anglaise où sa connaissance des textes romanesques unie à sa connaissance de la réalité sociologique des classes populaires en débusque plus d'un dans le roman réaliste comme dans le feuilleton ou la romance d'évasion. Ici le sociologue épaule utilement le critique : Hoggart est bien placé pour reconnaître au D. H. Lawrence d'Amants et fils, par contraste avec les romanciers populistes, ce ton de justesse à la fois littéraire et sociologique dont il est fin connaisseur puisque pratiquant subtil. Mais encore une fois, la justesse du ton hoggartien, qui fait son efficacité littéraire, me semble devoir d'abord son ressort expressif à la justice descriptive à laquelle est engagé le sociologue. Comment on acquiert cette disposition est une autre histoire dont l'autobiographie de Hoggart donne le récit sociologique, mais dont je ne dis pas que la sociologie qu'on peut en tirer épuise toute la sève. 


\section{NOTES}

*. Ce texte a été écrit en hommage à Raymonde Moulin, pour un volume de Mélanges. Il est issu d'une interview réalisée pour le Centre Georges Pompidou, en mars 1992. Les questions sont de Martine Chaudron, Chef du Service des Études et de la Recherche de la Bibliothèque Publique d'Information.

1. R. Hoggart, La culture du pauvre. Étude sur le style de vie des classes populaires en Angleterre, trad. fr. par F. et J.-C. Garcias, présentation et index de J.-C. Passeron, Paris, Minuit, 1970, 420 p. (1 $1^{\text {re }}$ éd. Londres, 1957). Dans l'accueil fait en France à ce livre, c'est Raymonde Moulin qui alerta les sociologues par le compte rendu qu'elle en donna, une des premières, dans la Revue Française de Sociologie (1971, vol. XII).

2. R. Hoggart, 33 Newport Street. Autobiographie d'un intellectuel issu des classes populaires anglaises, trad. fr. par C. et C. Grignon avec C. Todd, présentation et index de C. Grignon, Paris, Gallimard/Seuil, 1991, 288 p. (1 $1^{\text {re }}$ éd. Londres, 1988).

3. R. Hoggart, The Uses of Literacy. Aspects of working-class life with special reference to publications and entertainments, Londres, Chatto \& Windus, 1957.

4. J.-C. Passeron, Le raisonnement sociologique. L'espace non-poppérien du raisonnement naturel, Paris, Nathan, 1991, p. 207-225.

5. R. Barthes, « L'effet de réel », Communications, 11, 1968.

6. La « fonction référentielle (ou dénotative) du langage » ou l'« épaississement » du récit, qui définit le réalisme littéraire par ses effets métonymiques et par opposition à la métaphore, sont entendus ici au sens où $\mathrm{R}$. Jakobson utilise ces notions pour caractériser, parmi les fonctions possibles du langage, celle qu'il nomme « poétique » en ce qu'elle prend appui sur l'attention prêtée par l'émetteur et le récepteur aux signifiants du message en tant que tels : «Linguistique et poétique » (p. 218) ou « Deux aspects du langage et deux types d'aphasie » (p. 63-66) in Essais de linguistique générale, Paris, Minuit,

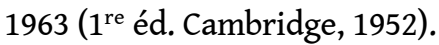

7. «L'illusion romanesque », in J.-C. Passeron, Le raisonnement sociologique..., p. 224.

8. R. Jakobson, « Du réalisme en art », in Questions de poétique, Paris, Seuil, 1973, p.. 34-39.

9. M. Riffaterre, «L'illusion référentielle ». Columbia Review, 57, 2, 1978.

10. «L'illusion romanesque », p. $x$.

11. Dans des romans comme Jude the Obscure de Thomas Hardy (1896) ou Félix Holt the Radical de George Eliot (1866), qu'Hoggart crédite d'ailleurs d'un certain réalisme romanesque qui lui semble disparaître aujourd'hui au profit de stéréotypes, « lorsque le sujet tombe dans les mains moins pures du journaliste ou du romancier à succès » (R. Hoggart, La culture du pauvre..., p. 40).

12. Pour reprendre le concept de Max Weber qui entend par là le produit de l'activité symbolique par laquelle tout groupe social satisfait le « besoin » de rendre cohérente l'expérience qu'il fait de sa condition, quelle qu'elle soit, en justifiant sa place dans l'univers considéré comme une totalité dotée de sens. On a là la définition wébérienne de la spécificité et de la fonctionnalité de ce qu'il appelle « culture ».

13. F. Venturi, Les Intellectuels, le Peuple et la Révolution. Histoire du populisme russe au XIXe siècle, Paris, Gallimard, 1972 (1 $1^{\text {re }}$ éd. Turin, 1952). 
14. P. Bourdieu et J.-C. Passeron, « Sociologues des mythologies et mythologies de sociologues », Les Temps Modernes, 24, 1963.

15. R. Hoggart, A Local Habitation. Life and Times : 1918-1940, Londres, Chatto \& Windus, 1988.

16. J'ai essayé d'analyser les facilités et les difficultés méthodologiques qui tiennent, pour l'historien comme pour le sociologue, au choix d'un ordre biographique de l'analyse dans «Le scénario et le corpus », Le raisonnement sociologique..., p. 185-206.

17. S. Settis, L'invention d'un tableau. «La tempête » de Giorgione, Paris, Minuit, 1987, « Le sujet caché », p. 123-156 ( $1^{\text {re }}$ éd. Turin, 1978).

18. Au sens wébérien où la sociologie commence avec l'effort d'associer ces deux intelligibilités. Économie et société, Paris, Plon, 1971, t. 1, p. 8-11 (1 ${ }^{\text {re }}$ éd. Tubingen, 1922). 19. Oserai-je soutenir, en versant au dossier ethnographique des rapports de la psychologie et de la sociologie un souvenir autobiographique, que la résistance des potaches des lycées français au discours professoral sur l'« âme humaine » (sur l'« actualité » et l'« universalité » des sentiments que décrit l'Iliade ou la tragédie classique) tient peut-être au fait que des enfants d'origine sociale différente possèdent encore (avant que l'endoctrinement par l'humanisme abstrait propre à l'École ait exercé sur eux tous ses effets de banalisation des essences), une conscience aiguë de l'insubstituabilité concrète des mondes affectifs et psychiques propres aux différents milieux sociaux, puisqu'ils expérimentent quotidiennement ces physionomies irréductibles du sentiment ou de l'émotion dans leurs familles respectives? J'ai constaté dans mes « humanités classiques » que, parmi les poncifs de l'enseignement des lettres, le postulat de l'« âme humaine » avait pour les potaches que nous étions, et surtout chez les moins bourgeois d'entre nous, une forte vertu comique par son irréalisme grandiloquent : nous n'avons pas dû être la seule classe de France à avoir défoulé notre scepticisme dans une orthographe gouailleuse : « la Mumaine ».

20. R. Hoggart, 33 Newport Street..., p. 138-139.

21. Ibid., p. 126-128.

22. Ibid., p. 195-201.

23. Ibid., p. 218-236.

24. Ibid., p. 217.

25. Ibid.

26. Ibid., p. 249-257, 270-280.

27. Ibid., p. 257.

28. C'est nous qui soulignons.

29. Ibid., p. 256.

30. C. Grignon et J.-C. Passeron, Le savant et le populaire. Misérabilisme et populisme en sociologie et en littérature, Paris, Le Seuil, 1989.

31. C'est de la comparaison entre l'altérité exotique et l'altérité populaire que s'est dégagé ce petit paradigme, que j'ai présenté d'abord dans « Le sens et la domination : la description du minimal » (Préface à La communication inégale, Paris, Éd. du CNRS, 1981), reproduit dans Le raisonnement sociologique..., p. 253-256.

32. La Lettre à une maîtresse d'école par les enfants de Barbiana (trad., Paris, Mercure de France, 1970 [1968] [1 ${ }^{\text {re }}$ éd. ital. 1963]) fournit un exemple presque pur de cet « oubli » populiste : le pamphlet - d'ailleurs bienvenu - qui oppose avec complaisance le recensement, en forme de faire-valoir, des savoir-faire des élèves issus des familles paysannes les plus pauvres (culture technique et rustique, vertus de caractère etc.) aux ridicules, aux maladresses et aux incompétences de leurs maîtres d'école, est obligé de 
constater, comme à regret, que la « relativisation » des critères de la valeur sociale reste une opération mentale sans influence sur l'ordre social : comme le dit un petit paysan à son institutrice petite-bourgeoise « On est donc quitte (chaque groupe a ses valeurs et ses critères) ; ou plutôt on le serait si chacun restait chez soi. Ou si vous aviez à passer des examens chez nous. Le malheur est que vous n'avez pas besoin de ça. » (p. 16).

33. Au sens de Chlovski pour qui le « procédé de singularisation » qui vise à « la libération de l'objet de l'automatisme perceptif » se retrouve dans toutes les formes de l'expression proprement littéraire ou artistique : V. Chlovski, « L'art comme procédé » (1929), trad. de T. Todorov in Théorie de la littérature, Paris, Seuil, 1965, en particulier p. 84-96. 